\title{
Cervical Spinal Cord and Dorsal Nerve Root Stimulation for Neuropathic Upper Limb Pain
}

\author{
Adrian B. Levine, Andrew G. Parrent, Keith W. MacDougall
}

\begin{abstract}
Background: Spinal cord stimulation (SCS) is a well-established treatment for chronic neuropathic pain in the lower limbs. Upper limb pain comprises a significant proportion of neuropathic pain patients, but is often difficult to target specifically and consistently with paresthesias. We hypothesized that the use of dorsal nerve root stimulation (DNRS), as an option along with SCS, would help us better relieve pain in these patients. Methods: All 35 patients trialed with spinal stimulation for upper limb pain between July 1, 2011, and October 31, 2013, were included. We performed permanent implantation in 23/35 patients based on a visual analogue scale pain score decrease of $\geq 50 \%$ during trial stimulation. Results: Both the SCS and DNRS groups had significant improvements in average visual analogue scale pain scores at 12 months compared with baseline, and the majority of patients in both groups obtained $\geq 50 \%$ pain relief. The majority of patients in both groups were able to reduce their opioid use, and on average had improvements in Short Form-36 quality of life scores. Complication rates did not differ significantly between the two groups. Conclusions: Treatment with SCS or DNRS provides meaningful long-term relief of chronic neuropathic pain in the upper limbs.
\end{abstract}

RÉSUMÉ: Stimulation de la moelle épinière et de la racine dorsale pour soulager la douleur neurogène des membres supérieurs. Contexte: La stimulation de la moelle épinière (SME) est un traitement de la douleur neurogène chronique des membres inférieurs qui a fait ses preuves. Une proportion importante de patients souffre aussi de douleur neurogène aux membres supérieurs mais il demeure ardu de cibler une telle douleur de façon systématique et spécifique en lien avec des manifestations de paresthésie. Nous avons ainsi formulé l'hypothèse que la stimulation de la racine dorsale, en plus de la SME, pourrait nous aider à mieux soulager la douleur chez ces patients. Méthodes: Tous les 35 patients chez qui on avait effectué, du $1^{\mathrm{er}}$ juillet 2011 au 31 octobre 2013, un traitement de SME des membres supérieurs ont été inclus dans cette étude. Pendant les essais cliniques de stimulation, nous avons soumis 23 patients sur 35 à un traitement continu. En nous fondant sur l'échelle visuelle analogique (ÉVA), nous avons anticipé une réduction de $\geq 50 \%$ du score lié à la douleur. Résultats: Au bout de 12 mois, tant les groupes ayant bénéficié de la SME que ceux ayant bénéficié de la stimulation de la racine dorsale ont obtenu, par rapport à des valeurs de référence, des scores nettement meilleurs en matière d'ÉVA de la douleur. En effet, une majorité de patients des deux groupes a rapporté un soulagement de la douleur de $\geq 50 \%$. Une majorité d'entre eux a aussi été en mesure de réduire sa consommation d'opiacés et a amélioré son score au test SF-36 (Short Form 36 Health Survey) en matière de qualité de vie. Fait à souligner, il n'y a pas eu de différence notable entre les deux groupes quant aux taux de complication. Conclusions: Tant la SME que la stimulation de la racine dorsale offrent un véritable soulagement à long terme de la douleur neurogène chronique des membres supérieurs.

Keywords: Neurosurgery, Pain, Spinal Cord, Chronic Pain, Pain Control, Spinal Cord Stimulation, Neuromodulation, Nerve Root Stimulation

doi:10.1017/cjn.2016.294

Can J Neurol Sci. 2017; 44: 83-89

Spinal cord stimulation (SCS) is a well-established treatment for chronic refractory neuropathic pain. There is a large body of evidence supporting its use, including several high-quality randomized controlled trials, mainly regarding stimulation of the thoracic spinal cord to treat lower limb pain resulting from failed back surgery syndrome ${ }^{1,2}$ and complex regional pain syndrome (CRPS). ${ }^{3,4}$ Some areas of the body are thought to be more difficult than others to cover consistently and specifically with paresthesias, such as the upper limbs, groin, and low back. ${ }^{5,6}$ Given that upper limb pain comprises a significant proportion of neuropathic pain patients, ${ }^{7}$ this is a population that has historically been undertreated with neuromodulation, and further study would likely result in significant improvements to their care.
Two important issues have been discussed in the literature regarding the placement of an electrode and stimulation of the cervical spinal cord. First, the spinal cord enlargement from C3 to C7, coupled with this region's susceptibility to degenerative changes and narrowing of the spinal canal, results in a shallow surrounding cerebrospinal space. ${ }^{5}$ This has raised concerns that

From the Department of Clinical Neurological Sciences, Division of Neurosurgery, Western University, London, ON, Canada.

Received July 9, 2015. Final Revisions Submitted May 8, 2016. Date of ACCEPTANCE July 8, 2016.

Correspondence to: Keith MacDougall, University Hospital, London Health

Sciences Centre, 339 Windermere Road, London, ON, Canada N6A 5A5.

Email: keith.macdougall@lhsc.on.ca. 
electrode placement in this region has a potentially increased risk of neurologic injury and other complications, compared to electrodes in the thoracic and lumbar spine. ${ }^{8}$ Second, variation of stimulation amplitude and coverage with body position changes is a common patient complaint with spinal stimulation. ${ }^{9}$ This issue is potentially exacerbated by the mobility of the cervical spine and can require awkward postures to maintain consistent stimulation, limiting the overall effectiveness of the device. ${ }^{5}$

Prior studies have reported success in treating upper limb CRPS and other forms of neuropathic pain with spinal cord stimulators. ${ }^{10-15}$ In our clinical practice, we have found that spinal dorsal nerve root stimulation (DNRS) is an effective technique for providing focused and consistent coverage for pain distributions and locations that are less amenable to treatment by traditional SCS. ${ }^{16,17}$ This technique uses the same stimulating electrodes and is easily converted to and from SCS during intraoperative testing, therefore it provides the surgeon with increased flexibility for targeting the pain area. In this paper, we report our experience with 35 patients treated for upper limb pain between July 1, 2011, to October 31, 2013, with a new trial of either SCS or DNRS.

\section{MeTHODS}

The study involved a single-center, open-label design and was approved by the Research Ethics Board at Western University, London, Canada. All patients who had been treated at University Hospital, London between July 1, 2011, and October 31, 2013, with a new implantation of a spinal stimulation trial system for upper limb pain entered the study.

\section{Patient Selection}

Patients were 18 years of age or older and had been referred for surgical management of upper limb pain that failed to respond to conservative measures including medication, psychological therapy, physical therapy, nerve blocks, and/or pain management programs. Exclusion criteria included pain in a distribution beyond the upper limb; another clinically significant or disabling chronic pain condition; an expected inability to manage or operate the SCS system; a history of a coagulation disorder; evidence of an active psychiatric disorder, another condition known to affect the perception of pain, or inability to evaluate treatment outcome; an existing or planned pregnancy; likelihood to undergo magnetic resonance imaging; and/or life expectancy of less than 1 year.

\section{Procedures}

Treatment involved a trial period of 3 weeks, during which the programming of the device was adjusted and the patient's response to the therapy was monitored. The trial electrodes were then removed and there was a mandatory washout period of at least two weeks. Permanent implantation followed successful trials, defined as a visual analogue scale (VAS) decrease $\geq 50 \%$ or a sufficiently large pain reduction to have a significant effect on the patient's quality of life. The stimulation technique was chosen was based the geographic distribution of the pain as well as intraoperative test stimulation results. In general, for the upper limb, nerve root stimulation was used in patients where the pain area was limited to one to four dermatomes. Electrodes were anchored to the thoracolumbar fascia using the standard technique with Boston Scientific's Clik anchors. Examples of the placement of SCS and DNRS electrodes in the cervical spine are shown in Figures 1 and 2, respectively.

\section{Data Collection and Analysis}

Average pain intensity was assessed on a $10 \mathrm{~cm}$ VAS in which the point furthest to the left represented no pain (score: 0 ) and that furthest to the right represented the worst possible pain (score: 10). Quality of life was assessed using the Short Form (SF)-36 questionnaire. These endpoints were assessed prospectively at the following time points: before trial stimulation, three weekly visits during the trial, before permanent implantation, at 3-, 6-, and 12-month follow-up visits, and annually thereafter. Evaluations were performed by the pain specialist neurosurgeon, a nurse trained in neuromodulation, or a research student. Results are reported at 12 months, with the exception of average VAS scores, which are plotted at 3-, 6-, and 12-month follow-up. Average values are presented as \pm standard error of the mean.

\section{Results}

\section{Patient Population and Trial Results}

There were a total of 35 eligible patients, of which 18 had trials for DNRS, 15 for SCS, and 2 for both DNRS and SCS. Three patients had multiple trials, two of which were to attempt different stimulation techniques, whereas one patient had to have his first trial operation aborted because of discomfort. Twenty-three patients $(66 \%)$ went on to permanent implantation, of which 11 received DNRS, $11 \mathrm{SCS}$, and one both.

Table 1 shows a breakdown of baseline characteristics for the SCS and DNRS groups of patients (the patients who received both were excluded from analysis because of small sample size). The two groups did not differ significantly in baseline VAS pain level, morphine equivalent daily dose (MEDD), SF-36 quality of life, employment status, age at implant, or time since pain onset. There was a greater proportion of female patients in the SCS group, of which $67 \%$ were female, compared with $44 \%$ of the DNRS patients. The most common pain diagnosis in each group was CRPS, followed by neuropathic pain not otherwise specified. The latter diagnosis included patients with posttraumatic, postsurgical, and spontaneous pain. There were two SCS patients with central neuropathic pain (poststroke and postspinal cord injury), and one DNRS patient with postherpetic neuralgia. All patients received electrodes placed in the cervical spine, and one DNRS patient also had an additional electrode in the thoracic spine (for both the trial and permanent implants).

\section{Pain Reduction}

Average VAS pain scores over 12 months of follow-up for each group of patients who received permanent implants are shown in Figure 3. At baseline, the average VAS was $7.8( \pm 1.2)$ for the SCS group and $7.6( \pm 1.2)$ for the DNRS group. At 12 months, the averages score had decreased to $2.7( \pm 0.6)$ for the SCS patients and $3.6( \pm 1.6)$ for the DNRS patients. At each of 3, 6 , and 12 months, significant improvements from baseline pain levels were seen for both groups, and there were no statistically significant differences between the two groups.

Patients were categorized as responders, partial responders, and nonresponders based on pain reduction of $\geq 50 \%, 30 \%$ to $50 \%$, and $<30 \%$, respectively. At 12 months, each patient group had pain data for nine patients, which is shown in Figure 4. In the 

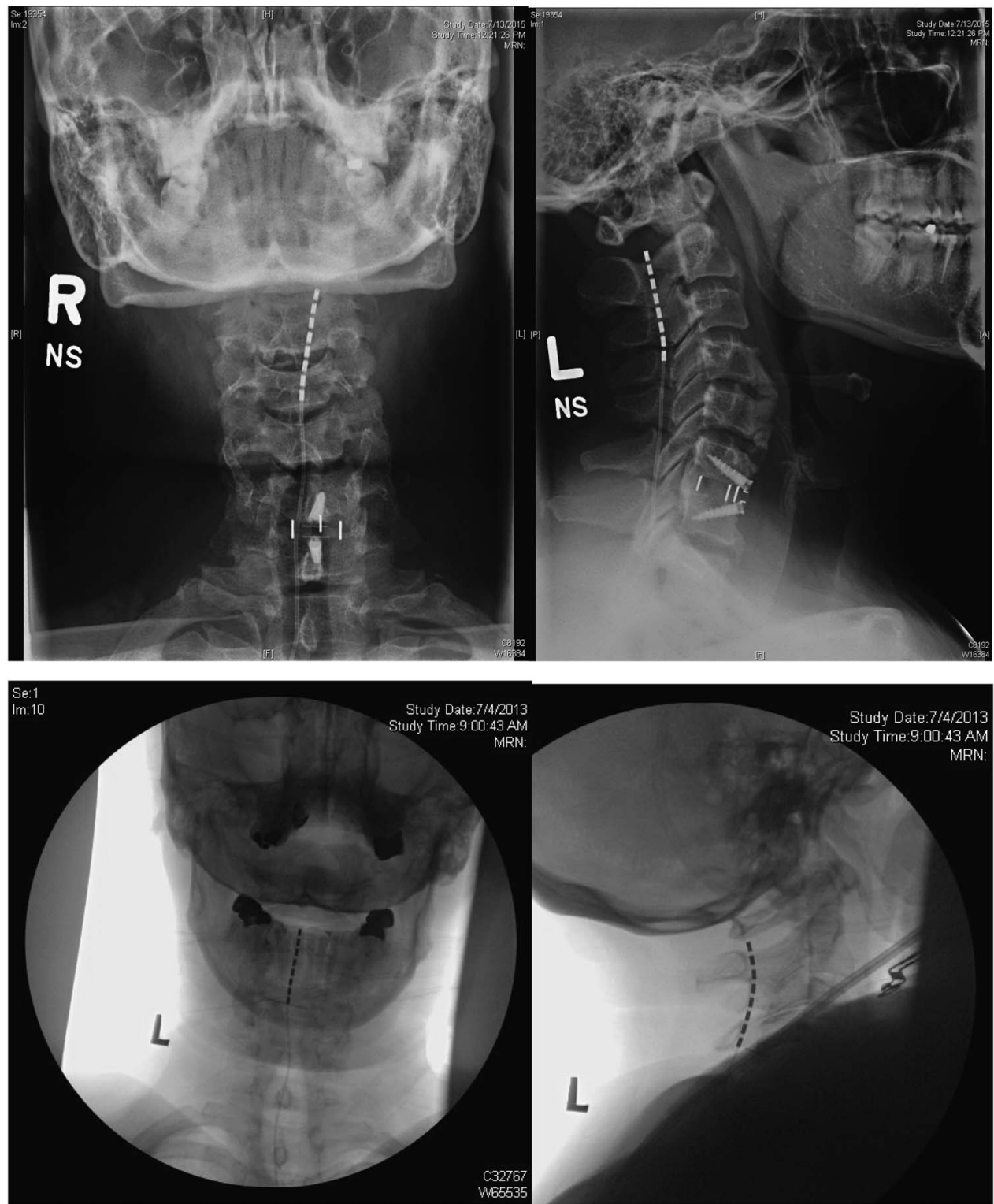

Figure 1: Anteroposterior and lateral X rays for two patients showing placement of SCS electrodes.

SCS group, six of nine (67\%) were responders, two (22\%) partial responders, and one (11\%) was a nonresponder. In the DNRS group, six of nine were responders $(67 \%)$ and three $(33 \%)$ were partial responders.

\section{Secondary Endpoints}

At baseline, both patient groups had large impairments in all components of the SF-36 questionnaire for health-related quality of life, without significant differences between the two groups. There were five SCS patients and four DNRS patients who had SF-36 scores available at both baseline and 12-month follow-up. Figure 5 shows the difference between 12-month scores and baseline scores for each group. Both patient groups had improvements in the average score for all nine components, with the DNRS patients generally having larger improvements. Because of the small sample size, confidence intervals were not included in the figure. The only score change that reached statistical significance was the physical functioning component for the SCS group.

Both groups of patients were taking large doses of opioids at baseline, with an average MEDD of $218 \mathrm{mg}( \pm 251)$ for SCS patients and $170 \mathrm{mg}( \pm 284)$ for DNRS patients. At 12 months, the average MEDD had decreased to $98 \mathrm{mg}( \pm 126)$ and $144 \mathrm{mg}$ $( \pm 259)$ for each group, respectively. Figure 6 shows the opioid doses at 12 months compared with baseline, divided into patients who had decreased, increased, and had no change in their dose, as well as patients who were never taking opioids. Of 10 SCS patients with data, five had decreased their MEDD, three had increased, and two were never on opioids. Of nine DNRS patients, six had decreased their MEDD and the remaining three were never on opioids.

At 12 months, five of six DNRS patients responded yes when asked if they were "satisfied with SCS treatment" and six of six 


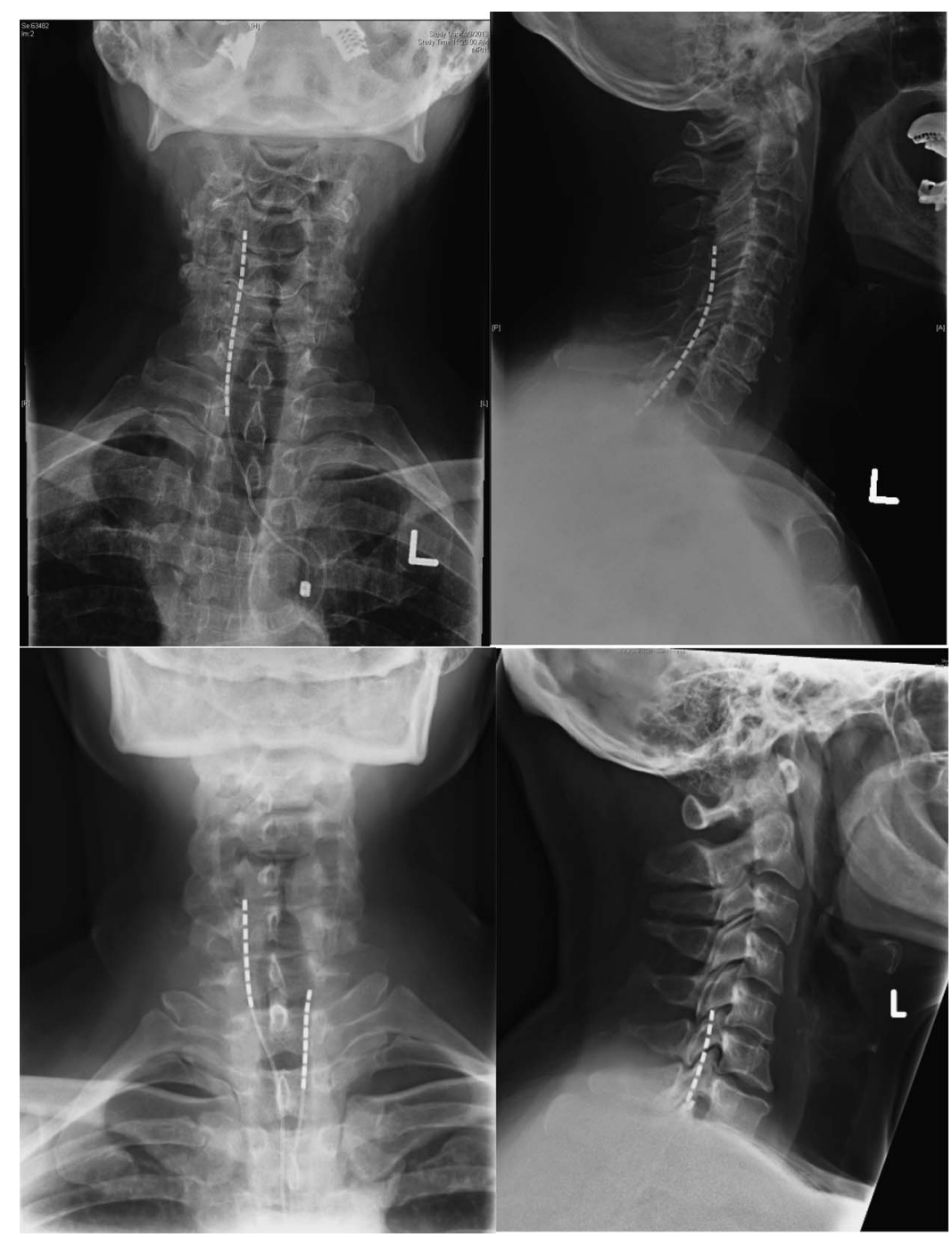

Figure 2: Anteroposterior and lateral X rays for three patients showing placement of DNRS electrodes.

responded yes to "recommend to a friend?" Among SCS patients, seven of seven responded yes to both questions. On a clinical global improvement scale on which 1 represented "very much improved" and 7 "very much worse," the average score was 1.75 $( \pm 0.71)$ for SCS patients and $1.67( \pm 1.03)$ for DNRS patients.

\section{Complications and Revision Operations}

During the trial period, the most common complications were superficial skin infection and lead migration, as shown in table 2. Infection occurred in two of 15 SCS patients (13\%) and three of 18 DNRS patients $(17 \%)$. One of these patients required surgical management of the infection, whereas the other four were treated just with oral antibiotics. Lead migration occurred in three of 15 (20\%) SCS patients and one of $18(6 \%)$ DNRS patients. Additionally, one SCS patient had to have a trial implant operation aborted because of discomfort, and one DNRS patient had a dural puncture during permanent implant, with resulting cerebrospinal leak and headache. During the permanent implant follow-up, there were a total of four revision operations for SCS patients and seven for DNRS patients. Three patients required multiple revision operations-one SCS patient had two revisions, one DNRS patient had two, and one DNRS patient had four. One SCS patient had his device removed because of lack of use. One DNRS patient had his electrode converted to an SCS system (he continued to be analyzed in the DNRS group). Otherwise, the reasons for revision were lead migration, implantable pulse generator malfunction, or attempted improvement of coverage.

\section{DISCUSSION}

Our study is the first to evaluate patients treated with dorsal nerve root stimulation of the cervical spine for upper limb pain. At 12 months, both the SCS and DNRS groups of patients had significant reductions in average VAS pain scores and the majority of patients obtained $\geq 50 \%$ pain reduction. In both groups, the majority of patients were able to reduce their opioid dose, and on average they had improvements in all subscores of the SF-36 questionnaire for quality of life. On patient satisfaction surveys, most patients indicated that they were satisfied with treatment and 
Table 1: Baseline patient characteristics

\begin{tabular}{|c|c|c|c|c|c|c|}
\hline & SCS & $N=15$ & $95 \% \mathrm{CI}$ & DNRS & $N=18$ & $95 \% \mathrm{CI}$ \\
\hline Age in years: mean, $\mathrm{SD}$ & 44.0 & 9.7 & 5.4 & 46.5 & 13.1 & 6.5 \\
\hline Years since pain onset: mean, SD & 7.1 & 4.2 & 2.3 & 5.3 & 2.5 & 1.3 \\
\hline Sex female: $\mathrm{n}, \%$ & 10 & 67 & & 8 & 44 & \\
\hline Currently employed: $\%$ & 43 & & & 35 & & \\
\hline VAS: mean, SD & 7.8 & 1.2 & 0.7 & 7.6 & 1.2 & 0.6 \\
\hline MEDD: mean, SD & 218 & 251 & 139 & 170 & 284 & 141 \\
\hline \multicolumn{7}{|l|}{ Diagnosis: $\mathrm{n}, \%$} \\
\hline CRPS & 9 & 60 & & 9 & 50 & \\
\hline Neuropathic not otherwise specified & 4 & 27 & & 8 & 44 & \\
\hline Central neuropathic & 2 & 13 & & 0 & 0 & \\
\hline Postherpetic neuralgia & 0 & 0 & & 1 & 6 & \\
\hline SF-36: mean, SD & \multicolumn{2}{|c|}{$\mathrm{N}=7$} & & \multicolumn{2}{|c|}{$\mathrm{N}=10$} & \\
\hline Physical functioning & 58 & 16 & 15 & 49 & 31 & 22 \\
\hline Role-physical & 4 & 9 & 9 & 16 & 36 & 24 \\
\hline Bodily pain & 19 & 21 & 19 & 18 & 18 & 12 \\
\hline General health & 70 & 18 & 16 & 48 & 25 & 17 \\
\hline Vitality & 35 & 14 & 13 & 27 & 20 & 13 \\
\hline Social functioning & 46 & 31 & 29 & 37 & 20 & 14 \\
\hline Role-emotional & 19 & 26 & 24 & 12 & 31 & 21 \\
\hline Mental health & 58 & 18 & 17 & 41 & 19 & 13 \\
\hline Health transition & 43 & 24 & 22 & 33 & 25 & 19 \\
\hline
\end{tabular}

$\mathrm{CI}=$ confidence interval $\mathrm{SD}=$ standard deviation.

would recommend it to a friend; the average clinical global impression was between "much improved" and "very much improved."

The technique for both SCS and DNRS at our centre uses the same hardware and stimulating electrodes. We must emphasize the need to place the electrode as far lateral as possible within the spinal canal for DNRS. Also, we take great care to ensure that the DNRS electrodes occupy the dorsal half of the neural foramina before stimulation to avoid ventral motor rootlet stimulation. Avoiding stimulation of the motor root also requires unique programming parameters. For dorsal root stimulation, we use a

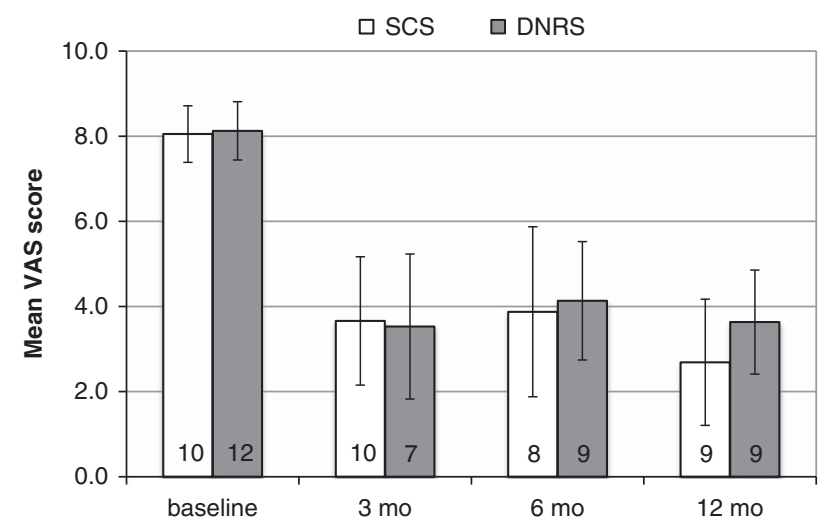

Figure 3: Aggregated average VAS pain scores for implanted patients over 12-month follow-up ( $N$ shown within bars). pulse width of 80 to $90 \mathrm{~ms}$, compared with 250 to 700 in the SCS patients. Amplitude for DNRS is generally less than $2 \mathrm{~mA}$. whereas it is highly variable for SCS.

Early in our experience, SCS was the initially trialed paradigm. Given current electrode technology, we can easily cover four cervical dermatomes/roots with DNRS. Therefore, if the pain area is confined to the C5-C8 dermatomes, we can cover the entire upper limb with DNRS. We have found DNRS to be much less positional overall and for upper limb pain it has become our default technique where possible. Although we did not specifically collect data regarding positional variations in stimulation amplitude and

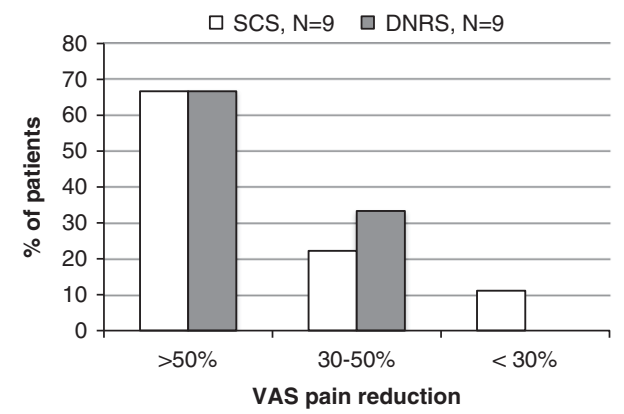

Figure 4: VAS pain reduction at 12 months, categorized as responders, partial responders, and nonresponders by $\%$ pain reduction from baseline value. 


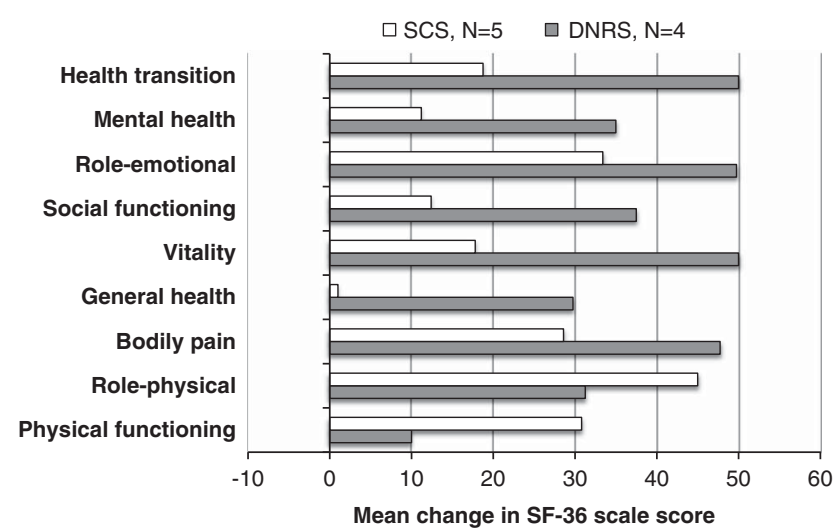

Figure 5: SF-36, change from baseline at 12 months.

effectiveness, anecdotally, when both techniques are tested in individual patients, they find DNRS much less variable during typical neck movements. Both surgeons have had the same experience and agree on this. Patients with upper limb pain radiating into the neck or scapula are generally trialed with SCS first.

The most common complications were lead migration and superficial skin infection (during the trial period with exposed leads). Taking into account the relatively small sample size in this article and potential for random variation in numbers, our complication rate did not vary significantly between the two groups of patients and was consistent with our rate for patients with stimulators at other spine levels as well as reported complication rates in the literature. There were more overall revision operations in the DNRS patient group; however, this figure is skewed by the fact that one patient required four revisions from repeated lead fractures and migration. During the trial period we had infections in five patients (one requiring surgical management) of 35 patients who received a total of 40 trial operations. Although our trial period of 3 weeks is longer than what is done at some other centres and can increase the risk of wound infection, we feel that a longer trial has value. We have found that there are patients who initially appear to be nonresponders who become successful candidates, as well as the opposite. It should also be noted that this subset of patients had a slightly higher infection rate than that of our entire database, which is below $10 \%$. Regarding

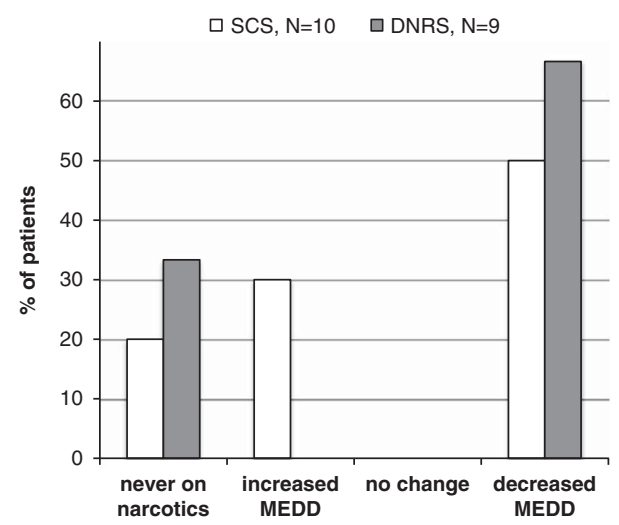

Figure 6: MEDD change at 12 months compared with initial assessment, categorized as patients who were never on narcotics, increased MEDD, decreased MEDD, or had no change in MEDD.
Table 2: Complications

\begin{tabular}{l|l|r|r|r|r}
\hline & & \multicolumn{2}{|c|}{ SCS } & \multicolumn{2}{c}{ DNRS } \\
\hline \multirow{3}{*}{ During trial period } & & $\mathbf{n}$ & $\%$ & $\mathbf{n}$ & $\%$ \\
\cline { 2 - 7 } & $\mathrm{N}$ & 15 & & 18 & \\
\cline { 2 - 7 } & Infection & 2 & 13 & 3 & 17 \\
\cline { 2 - 7 } & Lead migration & 3 & 20 & 1 & 6 \\
\cline { 2 - 7 } & CSF leak & 0 & 0 & 0 & 0 \\
\hline \multirow{5}{*}{ During permanent implant } & $\mathrm{N}$ & 11 & & 11 & \\
\cline { 2 - 7 } & CSF leak & 0 & 0 & 1 & 9 \\
\hline \multirow{2}{*}{ Revision operations } & Required 1 & 2 & 18 & 1 & 9 \\
\cline { 2 - 7 } & Required $>1$ & 1 & 9 & 2 & 18 \\
\cline { 2 - 7 } & Total number & 4 & & 7 & \\
\hline
\end{tabular}

$\mathrm{CSF}=$ cerebrospinal fluid.

the difference in trial period electrode migration rate between SCS (20\%) and DNRS (6\%) groups, we hesitate to draw any conclusions from such a small sample rate. That said, it is possible that the electrode is better anchored by the shape of the lateral epidural space and it does subjectively feel more secure during insertion.

Our study has a number of strengths. It includes a large number of consecutive patients in a clinical setting, with a broad range of pain diagnoses, compared with other studies that have a more homogenous patient sample. Our patients were referred by family doctors or pain specialists and had severe long-term pain that had failed medical therapy and other forms of treatment. We had no unusual exclusion criteria for upper limb patients other than our standard clinical criteria for appropriate neuromodulation patients (listed in the Methods section). For analysis, we have separated the patients with DNRS and traditional SCS. Our results indicate that, contrary to previous assertions that nerve root stimulation is not effective, this lesser known technique performs equally well in appropriately selected patients.

The main limitation is that this is a self-funded study with limited resources; therefore, not all data are obtained at every clinic visit. This was particularly apparent for the SF-36 questionnaire; of 22 patients receiving permanent implants, only nine had SF-36 data at both baseline and at 12 months, whereas 19 had VAS data at this time point. It would have been preferable to have an independent third party doing patient evaluations, but this was not feasible given our resources. Our patients were not randomized to treatment groups but were assigned based on which technique was expected to be more effective for the individual based on pain distribution and intraoperative testing.

Prior studies have been published that have obtained similar results in pain reduction. A retrospective study of 23 patients with cervical spinal cord stimulation published in 2012 found that average pain scores decrease from 6.8 to $2.8,{ }^{12}$ and a 2003 prospective study of 41 patients with either neuropathic or ischemic pain reported that $68 \%$ of patients obtain $>50 \%$ pain relief. ${ }^{10}$ A prospective study published in 2004 by Forouzanfar et al. evaluated 36 CRPS patients, 19 of whom were treated with electrodes placed in the cervical spine and 17 in the lumbar spine. As with our findings comparing our upper limb patients with those treated with stimulators in other locations, this study found no significant difference in either pain relief or complication rate between the two groups. 
An excellent paper was published by Deer et al. in 2014, outlining their prospective trial of 38 patients receiving cervical spinal cord stimulation, as well as a systematic literature review on the topic. ${ }^{13}$ Twelve studies were found that fulfilled their inclusion criteria, including a total of 221 patients. This included four prospective trials, four retrospective studies, and four case series. The most commonly cited adverse events were hardware malfunction $(17.8 \%)$, lead migration (13.9\%), and lead breakage (6.7\%). Despite the fears that the cervical spine could have greater risk of cord compression during implantation, there were no reports of neurologic damage in the 180 patients for whom adverse events were reported. This review found no significant difference in the complication rate for cervical electrodes compared with previously published rates for thoracic and lumbar electrodes.

In summary, these findings indicate that SCS and DNRS in the cervical spine can provide good pain relief in upper limb neuropathic pain, as well as enable opioid use reduction and improve health-related quality of life. Despite concerns that electrodes in the cervical spine could be more predisposed to complications than those at other levels, our complication rates in this study are consistent with those reported in the literature for both stimulation at the cervical spine and at other spinal levels. Most important, no patient developed a new neurological deficit during the study.

\section{Disclosures}

The authors have nothing to disclose.

\section{Statement of Authorship}

AL collected and entered data into the database, interpreted and analysed data, conducted a literature review, and wrote the manuscript. AP participated in development of the study and helped to evaluate and edit the manuscript. KM acted as corresponding author, supervised the study, interpreted data, and edited the manuscript.

\section{REFERENCES}

1. North RB, Kidd DH, Farrokhi F, Piantadosi SA. Spinal cord stimulation versus repeated lumbosacral spine surgery for chronic pain: a randomized, controlled trial. Neurosurgery. 2005;56:98-106.
2. Kumar K, Taylor RS, Jacques L, et al. Spinal cord stimulation versus conventional medical management for neuropathic pain: a multicentre randomised controlled trial in patients with failed back surgery syndrome. Pain. 2007;132:179-88.

3. Turner JA, Loeser JD, Deyo RA, Sanders SB. Spinal cord stimulation for patients with failed back surgery syndrome or complex regional pain syndrome: a systematic review of effectiveness and complications. Pain. 2004;108:137-47.

4. Kemler MA, Barendse GA, van Kleef M, et al. Spinal cord stimulation in patients with chronic reflex sympathetic dystrophy. N Engl J Med. 2000;343:618-24.

5. Levy RM. Anatomic considerations for spinal cord stimulation. Neuromodulation. 2014;17(Suppl 1):2-11.

6. Barolat G, Massaro F, He J, Zeme S, Ketcik B. Mapping of sensory responses to epidural stimulation of the intraspinal neural structures in man. J Neurosurg. 1993;78:233-9.

7. Torrance N, Smith BH, Bennett MI, Lee AJ. The epidemiology of chronic pain of predominantly neuropathic origin. Results from a general population survey. J Pain. 2006;7:281-9.

8. Falowski S, Ooi YC, Sabesan A, Sharan A. Spinal cord injury induced by a cervical spinal cord stimulator. Neuromodulation. 2011;14: 34-6.

9. Ross E, Abejon D. Improving patient experience with spinal cord stimulation: implications of position-related changes in neurostimulation. Neuromodulation. 2014;17(Suppl 1):36-41.

10. Simpson BA, Bassett G, Davies K, Herbert C, Pierri M. Cervical spinal cord stimulation for pain: a report on 41 patients. Neuromodulation. 2003;6:20-6.

11. Forouzanfar T, Kemler MA, Weber WE, Kessels AG, van Kleef M. Spinal cord stimulation in complex regional pain syndrome: cervical and lumbar devices are comparably effective. $\mathrm{Br} \mathrm{J}$ Anaesth. 2004;92:348-53.

12. Wolter T, Kieselbach K. Cervical spinal cord stimulation: an analysis of 23 patients with long-term follow-up. Pain Physician. 2012;15:203-12.

13. Deer TR, Skaribas IM, Haider N, et al. Effectiveness of cervical spinal cord stimulation for the management of chronic pain. Neuromodulation. 2014;17:265-71.

14. Chivukula S, Tempel ZJ, Weiner GM, et al. Cervical and cervicomedullary spinal cord stimulation for chronic pain: efficacy and outcomes. Clin Neurol Neurosurg. 2014;127:33-41.

15. Vallejo R, Kramer J, Benyamin R. Neuromodulation of the cervical spinal cord in the treatment of chronic intractable neck and upper extremity pain: a case series and review of the literature. Pain Physician. 2007;10:305-11.

16. Haque R, Winfree CJ. Spinal nerve root stimulation. Neurosurg Focus. 2006;21:E4.

17. Struijk JJ, Holsheimer J, Boom HB. Excitation of dorsal root fibers in spinal cord stimulation: a theoretical study. IEEE Trans Biomed Eng. 1993;40:632-9. 